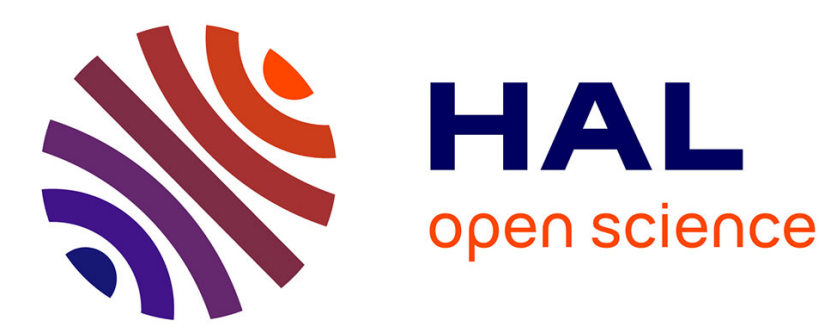

\title{
Minimizing decoherence on target in bipartite open quantum systems
}

\author{
Paolo Forni, Alain Sarlette
}

\section{To cite this version:}

Paolo Forni, Alain Sarlette. Minimizing decoherence on target in bipartite open quantum systems. CDC 2019 - 58th IEEE Conference on Decision and Control, Dec 2019, Nice, France. hal-02393976

\section{HAL Id: hal-02393976 \\ https://hal.inria.fr/hal-02393976}

Submitted on 4 Dec 2019

HAL is a multi-disciplinary open access archive for the deposit and dissemination of scientific research documents, whether they are published or not. The documents may come from teaching and research institutions in France or abroad, or from public or private research centers.
L'archive ouverte pluridisciplinaire HAL, est destinée au dépôt et à la diffusion de documents scientifiques de niveau recherche, publiés ou non, émanant des établissements d'enseignement et de recherche français ou étrangers, des laboratoires publics ou privés. 


\title{
Minimizing decoherence on target in bipartite open quantum systems
}

\author{
Paolo Forni ${ }^{1}$, Alain Sarlette ${ }^{2}$
}

\begin{abstract}
We consider a target quantum system, coupled to an auxiliary quantum system which dissipates rapidly at somewhat adjustable rates. The goal is to minimize the dissipation induced on the target system by this coupling. We use explicit model reduction formulas to express this as a quadratic optimization problem. We prove that maybe counterintuitively, when the auxiliary system dissipates along Hermitian (entropy-increasing) channels, the minimum induced dissipation is reached by maximizing the dissipation rate of the auxiliary system.
\end{abstract}

\section{INTRODUCTION}

The development of second-generation quantum technologies relies on an accurate control and stabilization of single quantum degrees of freedom, which encode quantum information. Considering such quantum systems as isolated from their environment is an idealized picture. Much of the difficulty in developing quantum processing hardware lies in achieving such controlled isolation in practice, at the very high accuracies necessary for obtaining the effects useful in applications.

Accordingly, one major aspect of building quantum hardware is to design a specific dominant behavior of the target system by smartly engineering some features of its environment. Belonging to this framework are two approaches. First, regarding the unavoidable coupling of a target system to some environment, one may focus on minimizing the perturbations, or decoherence, induced by this coupling on the information stored in the target system. This is the standard effort on isolating a system from perturbations. Second, by viewing part of the environment as a stabilizing controller, one would wish to maximize a particular stabilizing effect induced on the target by its coupling to the controller. This stabilization, usually called "reservoir engineering" [9], should be understood in the framework of information protection as irreversible operations associated to error-correcting codes [4]. Designing a system, out of any realistically available components, which would optimally process quantum information is the aim of building a viable quantum computer.

In the present paper we address a minimal part of this aim. Namely, we assume that a target system is given, with a fixed unavoidable coupling to a given dissipative environment, but the strength of the dissipation acting on the environment

\footnotetext{
${ }^{1}$ Centre Automatique et Systèmes, Mines-ParisTech, PSL Research University. 60 Bd Saint-Michel, 75006 Paris, France; and INRIA Paris, 2 rue Simone Iff, 75012 Paris, France. paolo.forni@inria.fr.

${ }^{2}$ INRIA Paris, 2 rue Simone Iff, 75012 Paris, France; and Ghent University / Electronics and Information Systems (ELIS), Technologiepark 914, 9052 Zwijnaarde, Belgium. alain.sarlette@inria.fr.

This work has been supported by the ANR project HAMROQS
}

can be adjusted. As a goal, we restrict ourselves to the first case, i.e. minimizing the impact of the environment on the target system. At first sight this may appear as a somewhat trivial situation: if adjustable, would you not just set all dissipations on the environment to their minimal value? The main motivation for this paper is that the answer to this question is no. Not only can some dissipation channels have a stabilizing effect on the environment, but even when channels encourage mixing in the environment, it can be better for the target system to make them stronger. In rough practical words, if your target system is weakly coupled to an environment that is subject to some unavoidable noise, then you can get a better system behavior by further increasing this noise - something that should not be too hard in practice.

Effects induced by the environment onto a target quantum system can be summarized in approximate reduced models, thanks to the timescale separation between the (weak) coupling of the environment to the target, and the rest of the dynamics. In particular, adiabatic elimination has been routinely used by physicists to eliminate fast dissipating components in composite quantum systems, and our analysis is based on the system-theoretic model reduction formulas of e.g. [1]. These assume that dissipation in the environment is much stronger than the coupling, and our conclusions thus remain valid when the dissipation can be adjusted within these limits in particular, it is perfectly compatible to require a maximal dissipation. Based on these adiabatic elimination expressions, we formulate the objective as a quadratic optimization problem. We then provide several analytic results about the optimal solution. In the case where all decoherence channels are Hermitian, which implies among others that they each tend to maximize entropy in the environment towards the fully mixed state, we prove that induced decoherence on the target is minimized when the strength of each decoherence channel on the environment is maximized. In other cases the situation can be more complicated, with some channels being maximized and others minimized, as we illustrate with simulation results on a standard qubit example.

The conclusion is linked to the fact that the decoherence channels do not act directly on the target system, but only through the dynamics of an environment. Apparently, inducing faster mixing in this environment somehow uncouples the dissipative effect from the target system. Thinking of rotating frames, this may be related to observations of dynamical decoupling [10], where a Hamiltonian actuation is set up with the explicit goal to decouple via averaging the target system from its environment. 


\section{Setting}

\section{A. Bipartite open quantum systems}

The object of our study is systems on the composite Hilbert space $\mathcal{H}:=\mathcal{H}_{A} \otimes \mathcal{H}_{B}$, composed of a target quantum system on $\mathcal{H}_{B}$ and its environment on $\mathcal{H}_{A}$. The dynamics on $\mathcal{H}$ is assumed to satisfy a time scale separation:

$$
\frac{d \rho}{d t}=\mathcal{L}_{A}(\rho)+\varepsilon \mathcal{L}_{\text {int }}(\rho)
$$

where $\varepsilon$ is a small positive parameter. $\mathcal{L}_{A}$ is a Lindbladian super-operator acting exclusively on $\mathcal{H}_{A}$. It comprises a Hamiltonian $\boldsymbol{H}_{A}$ and $K_{g}$ decoherence channels, whose strengths can be tuned by appropriately selecting some gain vector $(h, \boldsymbol{g}):=\left[h, g_{1}, \ldots, g_{K_{g}}\right]^{\top} \in \mathbb{R}_{\geqslant 0}^{K_{g}+1}$; plus $K_{e}$ decoherence channels whose strengths are fixed. Explicitly:

$\mathcal{L}_{A}^{h, \boldsymbol{g}}(\rho)=-i\left[h \boldsymbol{H}_{A}, \rho\right]+\sum_{k=1}^{K_{g}} g_{k} \mathcal{D}\left[\boldsymbol{L}_{k}\right](\rho)+\sum_{k=1}^{K_{e}} e_{k} \mathcal{D}\left[\boldsymbol{M}_{k}\right](\rho)$

with $\mathcal{D}\left[\boldsymbol{D}_{k}\right](\rho):=\boldsymbol{D}_{k} \rho \boldsymbol{D}_{k}^{\dagger}-\frac{1}{2}\left(\boldsymbol{D}_{k}^{\dagger} \boldsymbol{D}_{k} \rho+\rho \boldsymbol{D}_{k}^{\dagger} \boldsymbol{D}_{k}\right)$, and the $\boldsymbol{L}_{k}, \boldsymbol{M}_{k}$ are given (not necessarily Hermitian) operators acting on $\mathcal{H}_{A}$. The interaction superoperator $\mathcal{L}_{\text {int }}$ is here assumed of Hamiltonian form and expressed as:

$$
\mathcal{L}_{i n t}(\rho):=-i\left[\sum_{j=1}^{J} \boldsymbol{A}_{j} \otimes \boldsymbol{B}_{j}^{\dagger}, \rho\right],
$$

where $\boldsymbol{A}_{j}$ and $\boldsymbol{B}_{j}$ are (not necessarily Hermitian) operators acting respectively on $\mathcal{H}_{A}$ and on $\mathcal{H}_{B}$ only.

We will specify in Section III-A that the gain vector $(h, \boldsymbol{g})$ takes values that always make $\mathcal{L}_{A}$ strongly dissipative with respect to the $\varepsilon$ timescale. Furthermore, $\mathcal{L}_{A}$ is assumed to admit a unique steady state $\bar{\rho}_{A}$ such that $\mathcal{L}_{A}\left(\bar{\rho}_{A}\right)=0$. For $\varepsilon=0$, the system is uncoupled and the solutions stay separable in $\mathcal{H}_{A}$ and $\mathcal{H}_{B}$ at all times.

\section{B. Adiabatic elimination formulas}

In this section, we recall a system-theoretic approach to adiabatic elimination and associated formulas for the class of systems (1)-(3), see [1] for more explanation. For $\varepsilon=0$, the system features an asymptotically stable center manifold $\mathcal{M}_{0}$, actually a subspace, of the same dimension as $\mathcal{H}_{B}$. By Fenichel's Invariant Manifold Theorem [5], for $0<\varepsilon \ll 1$ small enough, there persists an asymptotically stable manifold $\mathcal{M}_{\varepsilon}$ which is $\varepsilon$-close to $\mathcal{M}_{0}$ and on which the dynamics is slow. Identifying this manifold and the dynamics on it is a systematic approach to eliminating the (uninteresting) fast dynamics of the environment, routinely called adiabatic elimination by physicists.

Let the manifold $\mathcal{M}_{\varepsilon}$ be the image of a parameter space $\operatorname{End}\left[\mathcal{H}_{s}\right]$ of dimension $\operatorname{dim} \operatorname{End}\left[\mathcal{H}_{B}\right]$ through a linear map $\mathcal{K}_{\varepsilon}: \operatorname{End}\left[\mathcal{H}_{s}\right] \rightarrow \operatorname{End}[\mathcal{H}]$. The dynamics on $\mathcal{M}_{\varepsilon}$ would be described by some linear operator $\mathcal{L}_{s, \varepsilon}$ on $\operatorname{End}[\mathcal{H}]$. Invariance of $\mathcal{M}_{\varepsilon}$ is then characterized by the following equation:

$$
\mathcal{L}_{A}\left(\mathcal{K}_{\varepsilon}\left(\rho_{s}\right)\right)+\varepsilon \mathcal{L}_{\text {int }}\left(\mathcal{K}_{\varepsilon}\left(\rho_{s}\right)\right)=\mathcal{K}_{\varepsilon}\left(\mathcal{L}_{s, \varepsilon}\left(\rho_{s}\right)\right)
$$

Following Carr's approach [2], operators $\mathcal{K}_{\varepsilon}$ and $\mathcal{L}_{s, \varepsilon}$ can be parameterized as infinite series

$$
\mathcal{K}_{\epsilon}\left(\rho_{s}\right)=\sum_{\ell=0}^{+\infty} \epsilon^{\ell} \mathcal{K}_{\ell}\left(\rho_{s}\right), \quad \mathcal{L}_{s, \epsilon}\left(\rho_{s}\right)=\sum_{\ell=0}^{+\infty} \epsilon^{\ell} \mathcal{L}_{s, \ell}\left(\rho_{s}\right) .
$$

By plugging (5) into (4) and identifying the terms of same order, an invariance relation is obtained at all orders.

To preserve the quantum structure of the reduced model, the authors in [1] conjectured that $\mathcal{K}_{\varepsilon}$ and $\mathcal{L}_{s, \varepsilon}\left(\rho_{s}\right)$ respectively take $\mathrm{Kraus}^{1}$ and Lindblad form at all orders of approximation. In particular, the main result in [1] proves that the conjecture is valid up to third-order terms.

We now recall the main result from [1] that is adopted in the remainder of this paper. Let $\boldsymbol{F}_{j}$ be a family of operators such that:

$\mathcal{L}_{A}\left(\boldsymbol{F}_{j} \bar{\rho}_{A}\right)+\boldsymbol{A}_{j} \bar{\rho}_{A}-\operatorname{Tr}\left[\boldsymbol{A}_{j} \bar{\rho}_{A}\right] \bar{\rho}_{A}=0, \quad \operatorname{Tr}\left[\boldsymbol{F}_{j} \bar{\rho}_{A}\right]=0$.

Existence and uniqueness of solutions of (6) can be proved along the lines of [1, Lemma 4]. Furthermore, let:

$$
\begin{aligned}
X_{k, j} & =\operatorname{Tr}\left[\boldsymbol{F}_{j} \bar{\rho}_{A} \boldsymbol{A}_{k}^{\dagger}+\boldsymbol{A}_{j} \bar{\rho}_{A} \boldsymbol{F}_{k}^{\dagger}\right] \\
Y_{k, j} & =\frac{1}{2 i} \operatorname{Tr}\left[\boldsymbol{F}_{j} \bar{\rho}_{A} \boldsymbol{A}_{k}^{\dagger}-\boldsymbol{A}_{j} \bar{\rho}_{A} \boldsymbol{F}_{k}^{\dagger}\right] .
\end{aligned}
$$

One shows (see [1, Lemma 7]) that matrix $X$ is Hermitian and positive-definite, thus there exists $\Lambda$ such that $X=\Lambda \Lambda^{\dagger}$. Furthermore, matrix $Y$ is Hermitian.

Theorem 1: [1, Section 3]: Consider model (1)-(3). The invariance condition resulting from $(4),(5)$ is satisfied up to second order by the Lindblad reduced dynamics:

$$
\begin{aligned}
\mathcal{L}_{s, \varepsilon}= & -i\left[\varepsilon \boldsymbol{H}_{s, 1}+\varepsilon^{2} \boldsymbol{H}_{s, 2}, \rho_{s}\right] \\
& +\varepsilon^{2} \sum_{p} \mathcal{D}\left[\boldsymbol{L}_{p}\right]\left(\rho_{s}\right)+\mathcal{O}\left(\varepsilon^{3}\right),
\end{aligned}
$$

with $\boldsymbol{H}_{s, 1}:=\sum_{k} \operatorname{Tr}\left[\boldsymbol{A}_{k} \bar{\rho}_{A}\right] \boldsymbol{B}_{k}^{\dagger}, \quad \boldsymbol{H}_{s, 2}:=\sum_{k, j} Y_{k, j} \boldsymbol{B}_{k} \boldsymbol{B}_{j}^{\dagger}$, and $\boldsymbol{L}_{p}=\sum_{j} \Lambda_{j, p}^{\star} \boldsymbol{B}_{j}^{\dagger}$; this dynamics is associated to a completely positive map $\mathcal{K}_{\varepsilon}\left(\rho_{s}\right)$ which is trace-preserving up to third-order terms.

Note that in this result, the decoherence channels induced on the target system come from coupling channels in the original system.

Remark 1: Theorem 1 shows that the Hamiltonian coupling to the environment $\mathcal{H}_{A}$ induces decoherence on the target $\mathcal{H}_{B}$ only at second order. Because matrix $X$ is positive semidefinite, the induced decoherence will be bounded by the diagonal elements of $\boldsymbol{X}$, i.e. by $\operatorname{Tr}\left[\boldsymbol{A}_{j}^{\dagger} \boldsymbol{R}_{j}+\boldsymbol{R}_{j}^{\dagger} \boldsymbol{A}_{j}\right]$ for $j \in\{1, \ldots J\}$, where $\boldsymbol{R}_{j}:=\boldsymbol{F}_{j} \bar{\rho}_{A}$. We will thus use the diagonal of $X$ as a measure to summarize the induced decoherence.

Remark 2: Model (1) assumes no dissipation on $\mathcal{H}_{B}$. If such dissipation $\mathcal{L}_{B}$ was present with strength $\varepsilon^{2}$, all our results still hold, with the induced dissipation just adding to $\mathcal{L}_{B}$. If $\mathcal{L}_{B}$ was of order $\varepsilon$, the same results would hold

\footnotetext{
${ }^{1}$ A Kraus map takes the form $\mathcal{K}\left(\rho_{s}\right)=\sum_{l} \boldsymbol{M}_{l} \rho_{s} \boldsymbol{M}_{l}^{\dagger}$ for some operators $\boldsymbol{M}_{l}$, to ensure complete positivity [3], with $\sum_{l} \boldsymbol{M}_{l}^{\dagger} \boldsymbol{M}_{l}=$ identity for trace preservation.
} 
provided a Hamiltonian control is added to cancel $\mathcal{H}_{s, 1}$ to first order; we cannot claim though that this would imply the best system protection; information encoding will have to be investigated explicitly as $\mathcal{L}_{B}$ would be dominating the induced dissipation by an order of magnitude $1 / \varepsilon$. For a similar reason, we did not investigate the case where $\mathcal{L}_{B}$ is of the same order as $\mathcal{L}_{A}$.

\section{General RESUlts}

\section{A. Formulation as optimization and time rescaling}

The aim of this work is to minimize the induced decoherence on the reduced quantum system according to $\mathcal{L}_{s, \varepsilon}$ from Theorem 1. We will assume that the coupling strength $\varepsilon$ is fixed, the decoherence channels acting on the environment are fixed too, but their strengths can be adapted within some range - in particular, the noise acting on the environment can be increased (at little practical cost).

In this section we set up the basic elements. We first reformulate decoherence minimization as a standard optimization problem and then observe the most trivial effect, namely overall rescaling.

To this aim, let the gains $h$ and $g_{k}$ for $k \in\left\{1, \ldots, K_{g}\right\}$ take values on a polytope $\Omega$ which is the product of strictly positive closed intervals, i.e.

$$
\Omega:=[\underline{h}, \bar{h}] \times \underset{k=1}{X_{g}}\left[\underline{q}_{k}, \bar{g}_{k}\right],
$$

with $g_{k}>0$ for all $k \in\left\{1, \ldots, K_{g}\right\}$. Most importantly, we must assume that $\boldsymbol{g}:=\left[q_{1}, \ldots, q_{K_{g}}\right]$ and coefficients $e_{k}$, $k \in\left\{1, \ldots, K_{e}\right\}$ together are large enough to guarantee the timescale separation of $\mathcal{L}_{A}^{h, g}$ with the environment, i.e. such that the spectral gap $\lambda_{s p}$ of $\mathcal{L}_{A}^{h, g}$ satisfies $\left|\lambda_{s p}\right| \gg \varepsilon$.

Minimizing the decoherence induced on the target system, according to $\mathcal{L}_{s, \varepsilon}$ from Theorem 1 , can be reformulated as the following quadratic optimization problem:

$$
\begin{array}{ll}
\min _{\boldsymbol{x}} t & \\
\text { s.t. } & \operatorname{Tr}\left[\boldsymbol{A}_{j}^{\dagger} \boldsymbol{R}_{j}+\boldsymbol{R}_{j}^{\dagger} \boldsymbol{A}_{j}\right] \leqslant t \\
& \operatorname{Tr}\left[\boldsymbol{R}_{j}\right]=0 \\
& \mathcal{L}_{A}^{h, \boldsymbol{g}}\left(\boldsymbol{R}_{j}\right)+\boldsymbol{A}_{j} \bar{\rho}_{A}-\operatorname{Tr}\left[\boldsymbol{A}_{j} \bar{\rho}_{A}\right] \bar{\rho}_{A}=0 \\
& \forall j \in\{1, \ldots, J\} \\
& \operatorname{Tr}\left[\bar{\rho}_{A}\right]=1 \\
& \mathcal{L}_{A}^{h, \boldsymbol{g}}\left(\bar{\rho}_{A}\right)=0 \\
& \underline{h} \leqslant h \leqslant \bar{h} \\
& \boldsymbol{g} \leqslant \boldsymbol{g} \leqslant \overline{\boldsymbol{g}},
\end{array}
$$

where $\boldsymbol{x}:=\left\{\boldsymbol{R}_{1}, \ldots, \boldsymbol{R}_{J}, \bar{\rho}_{A}, h, \boldsymbol{g}, t\right\}$ represents the optimization variables. Statement $(9 \mathrm{a})-(9 \mathrm{~b})$ corresponds to the objective of minimizing the diagonal elements of $\boldsymbol{X}$, as mentioned in Remark 1. Equality constraints (9d)-(9e) correspond to (6), whereas (9e)-(9f) express that $\bar{\rho}_{A}$ is the steadystate density operator of $\mathcal{L}_{A}^{g}$.

Note that the factorization of $\boldsymbol{A}_{j} \otimes \boldsymbol{B}_{j}^{\dagger}$ is only defined up to a scalar for each $j$. Therefore, when assigning the same bound for each $j$ in (9b), we implicitly assume that these scalars have been chosen such that a given decoherence speed takes the same practical importance for each channel $j$. A more complicated treatment of the relation between different channels is of course possible along the same principles, including the off-diagonals of $X$ as well; we just take this form (9b) for concreteness. In further analysis we will assume a single coupling channel, so these questions will not appear.

A first observation on (9) is the following result.

Proposition 1: Consider problem (9) and assume that $K_{e}=0$. Then, for the optimizer $\boldsymbol{x}^{\star}=$ $\left\{\boldsymbol{R}_{1}^{\star}, \ldots, \boldsymbol{R}_{J}^{\star}, \bar{\rho}_{A}^{\star}, h^{\star}, \boldsymbol{g}^{\star}, t^{\star}\right\}$, either we have $h^{\star}=\bar{h}$ or there exists at least an index $\bar{k}$ such that $\boldsymbol{g}_{\bar{k}}^{\star}=\overline{\boldsymbol{g}}_{\bar{k}}$.

Proof: Assume by contradiction that $\boldsymbol{g}^{\star}<\overline{\boldsymbol{g}}$ and $h^{\star}<$

$\bar{h}$. Then there exists a constant

$$
\alpha:=\max \left\{r \geqslant 0 \text { s.t. } r \boldsymbol{g}^{\star} \leqslant \overline{\boldsymbol{g}} \text { and } \alpha h^{\star} \leqslant \bar{h}\right\}>1 .
$$

One checks that $\left\{\alpha^{-1} \boldsymbol{R}_{1}^{\star}, \ldots, \alpha^{-1} \boldsymbol{R}_{J}^{\star} \bar{\rho}_{A}^{\star}, \alpha h^{\star}, \alpha \boldsymbol{g}^{\star}, \alpha^{-1} t^{\star}\right\}$ is a better minimizer than $\boldsymbol{x}^{\star}$ for (9).

Remark 3: $\alpha$ expresses time-rescaling. If $K_{e} \neq 0$, the solely feasible time-rescaling is the trivial one, $\alpha=1$.

Proposition 1 provides the maybe counter-intuitive result that adiabatic elimination reverses the time scaling: the faster the environment dissipates, the slower the reduced-order model decoheres, and viceversa. We will briefly discuss the interpretation of this effect in the conclusion.

\section{B. Single interaction channel and mixing decoherence}

We have so far only looked at rescaling the speed of motion of the environment treated as a whole. A more interesting question is how we may want to tune each individual decoherence channel. Quantum decoherence channels can be of different types. A channel like $\boldsymbol{L}_{k}=\boldsymbol{\sigma}_{-}=|g\rangle\langle e|$ for a qubit, expressing spontaneous photon emission, is in fact stabilizing the state towards a well-defined value (zero entropy). Once this would be done, the target system only sees a well-defined environment state, where the decoherence dynamics annihilates, and it would not be surprising that the target system thus behaves well.

In contrast, a Hermitian channel like $\boldsymbol{L}_{k}=|g\rangle\langle g|-| e\rangle\langle e|$ for a qubit, expressing dephasing between energy levels, always pushes the environment towards a more entropic state, or in other words "mixes" its state. In this section, we obtain results for such mixing environments, whose entropy can only increase in time, under the technical simplifying assumption of a single Hamiltonian interaction term. We show (see Corollary 1) that, maybe surprisingly, decoherence induced on the target system again is minimized when the strength of each mixing channel in the environment is maximized.

Let $J=1$ and thus:

$$
\mathcal{L}_{\text {int }}(\rho)=-i[\boldsymbol{A} \otimes \boldsymbol{B}, \rho],
$$

where $\boldsymbol{A}$ and $\boldsymbol{B}$ respectively are Hermitian operators acting on $\mathcal{H}_{A}$ and $\mathcal{H}_{B}$ only. The class of environments in consideration is characterized by the following assumptions. 
Assumption 1: The tunable decoherence operators in $\mathcal{L}_{A}^{g}$ are Hermitian, i.e. $\boldsymbol{L}_{k}^{\dagger}=\boldsymbol{L}_{k}$ for all $k \in\left\{1, \ldots, K_{g}\right\}$, and $h=0$.

Assumption 2: For some $\hat{\boldsymbol{g}} \in[\boldsymbol{g}, \overline{\boldsymbol{g}}]$, the unique steadystate featured by $\mathcal{L}_{A}^{\hat{g}}$ is $\bar{\rho}_{A}=\boldsymbol{I} / n$ where $n:=\operatorname{dim}\left[\mathcal{H}_{A}\right]$.

Assumption 2 involves the $K_{e}$ fixed decoherence channels. It is satisfied e.g. for a qubit subject to relaxation in a thermal environment in the limit where the average number $n_{t h}$ of thermal photons is infinite: having two fixed channels $M_{1}=$ $\sqrt{\gamma\left(1+n_{t h}\right)} \boldsymbol{\sigma}_{-}$and $\boldsymbol{M}_{2}=\sqrt{\gamma n_{t h}}\left(\boldsymbol{\sigma}_{-}\right)^{\dagger}$, taking the limit $n_{t h} \rightarrow+\infty$ with $\gamma n_{t h}$ finite we have $\bar{\rho}_{A}=\boldsymbol{I} / 2$.

Under Assumptions 1 and $2, \bar{\rho}_{A}=\boldsymbol{I} / n$ remains the unique steady-state of $\mathcal{L}_{A}^{\boldsymbol{g}}$ for any $\boldsymbol{g} \leqslant \boldsymbol{g} \leqslant \overline{\boldsymbol{g}}$. Therefore the optimization problem (9) simplifies to:

$$
\begin{array}{ll}
\min _{\boldsymbol{x}} & \operatorname{Tr}[\boldsymbol{A} \boldsymbol{F}] \\
\text { s. t. } & \operatorname{Tr}[\boldsymbol{F}]=0 \\
& \mathcal{L}_{A}^{\boldsymbol{g}}(\boldsymbol{F})+\boldsymbol{A}-\frac{1}{n} \operatorname{Tr}[\boldsymbol{A}] \boldsymbol{I}=0 \\
& \boldsymbol{g} \leqslant \boldsymbol{g} \leqslant \overline{\boldsymbol{g}}
\end{array}
$$

where $\boldsymbol{x}:=\{\boldsymbol{F}, \boldsymbol{g}\}$ represents the optimization variables. The Lagrangian for (11) reads as:

$$
\begin{aligned}
\mathfrak{L}(\tilde{\boldsymbol{x}})= & \operatorname{Tr}[\boldsymbol{A} \boldsymbol{F}]+\sum_{k=1}^{K_{g}} \underline{\mu}_{k}\left(-g_{k}+g\right)+\bar{\mu}_{k}\left(g_{k}-\bar{g}\right) \\
& +\lambda_{\text {trace }} \operatorname{Tr}[\boldsymbol{F}] \\
& +\operatorname{Tr}\left[\boldsymbol{\Lambda}_{\text {inv }}\left(\mathcal{L}_{A}^{\boldsymbol{g}}(\boldsymbol{F})+\boldsymbol{A}-\frac{1}{n} \operatorname{Tr}[\boldsymbol{A}] \boldsymbol{I}\right)\right]
\end{aligned}
$$

where $\tilde{\boldsymbol{x}}:=\left\{\boldsymbol{F}, \boldsymbol{g}, \lambda_{\text {trace }}, \boldsymbol{\Lambda}_{\text {inv }}, \underline{\boldsymbol{\mu}}, \overline{\boldsymbol{\mu}}\right\}$ while $\lambda_{\text {trace }}, \boldsymbol{\Lambda}_{\text {inv }}, \underline{\boldsymbol{\mu}}$, and $\overline{\boldsymbol{\mu}}$ respectively are a scalar, a matrix, and two vectors of Karush-Kuhn-Tucker multipliers on the equality and inequality constraints.

The Karush-Kuhn-Tucker necessary conditions for the optimizer $\tilde{\boldsymbol{x}}^{\star}$ of (11) read as:

$$
\begin{aligned}
& 0=\boldsymbol{A}+\lambda_{\text {trace }}^{\star} \boldsymbol{I}+\sum_{k=1}^{K_{g}} g_{k}^{\star} \mathcal{D}\left[\boldsymbol{L}_{k}\right]\left(\boldsymbol{\Lambda}_{\text {inv }}^{\star}\right) \\
& 0=-\underline{\boldsymbol{\mu}}^{\star}+\overline{\boldsymbol{\mu}}^{\star}+\left(\begin{array}{c}
\operatorname{Tr}\left[\boldsymbol{\Lambda}_{\text {inv }}^{\star} \mathcal{D}\left[\boldsymbol{L}_{1}\right]\left(\boldsymbol{F}^{\star}\right)\right] \\
\vdots \\
\operatorname{Tr}\left[\boldsymbol{\Lambda}_{i n v}^{\star} \mathcal{D}\left[\boldsymbol{L}_{K}\right]\left(\boldsymbol{F}^{\star}\right)\right]
\end{array}\right) \\
& 0=\overline{\boldsymbol{\mu}}^{\star} \odot\left(\boldsymbol{g}^{\star}-\overline{\boldsymbol{g}}\right) \\
& 0=\boldsymbol{\mu}^{\star} \odot\left(-\boldsymbol{g}^{\star}+\boldsymbol{g}\right) \\
& 0 \leqslant \overline{\boldsymbol{\mu}}^{\star} \\
& 0 \leqslant \boldsymbol{\mu}^{\star}
\end{aligned}
$$

where $\odot$ denotes element-wise multiplication.

Proposition 2: If $\tilde{\boldsymbol{x}}_{1}^{\star}=\left\{\boldsymbol{F}^{\star}, \boldsymbol{g}^{\star}, \lambda_{\text {trace }}^{\star}, \boldsymbol{\Lambda}_{\text {inv }}^{\star}, \underline{\boldsymbol{\mu}}^{\star}, \overline{\boldsymbol{\mu}}^{\star}\right\}$ is a minimizer for (11) satisfying $g_{\bar{k}}^{\star} \in\left(g_{\bar{k}}, \bar{g}_{\bar{k}}\right)$ for some $\bar{k}$, then $\tilde{\boldsymbol{x}}_{2}^{\star}:=\left\{\boldsymbol{F}^{\star}, \boldsymbol{h}, \lambda_{\text {trace }}^{\star}, \boldsymbol{\Lambda}_{\text {inv }}^{\star}, \underline{\boldsymbol{\mu}}^{\star}, \overline{\boldsymbol{\mu}}^{\star}\right\}$ with $h_{k}=g_{k}^{\star}$ for all $k \neq \bar{k}$ and any $h_{\bar{k}} \in\left[g_{\bar{k}}, \bar{g}_{\bar{k}}\right]$ is also a minimizer for (11) attaining the same minimum of the cost function.

Proof: Slackness conditions (13c)-(13f) immediately imply that $\mu_{\bar{k}}^{\star}=\bar{\mu}_{\bar{k}}^{\star}=0$ and thus $0=\overline{\boldsymbol{\mu}}^{\star} \odot(\boldsymbol{h}-\overline{\boldsymbol{g}})=$ $\underline{\boldsymbol{\mu}}^{\star} \odot(-\boldsymbol{h}+\boldsymbol{g})$ and $\overline{\boldsymbol{\mu}}^{\star}, \boldsymbol{\mu}^{\star} \geqslant 0$. By (13b), we then have $\operatorname{Tr}\left[\boldsymbol{\Lambda}_{i n v}^{\star} \mathcal{D}\left[\boldsymbol{L}_{\bar{k}}\right]\left(\boldsymbol{F}^{\star}\right)\right]=0$. By Lemmas 1 and 2 in appendix, we then have $\mathcal{D}\left[\boldsymbol{L}_{\bar{k}}\right]\left(\boldsymbol{F}^{\star}\right)=0$ and thus

$$
\begin{aligned}
\mathcal{L}_{A}^{\boldsymbol{g}^{\star}\left(\boldsymbol{F}^{\star}\right)} & =\mathcal{L}_{A}^{\boldsymbol{g}^{\star}}\left(\boldsymbol{\Lambda}_{i n v^{\star}}\right)=\sum_{k \neq \bar{k}} g_{k}^{\star} \mathcal{D}\left[\boldsymbol{L}_{k}\right]\left(\boldsymbol{\Lambda}_{\text {inv }}^{\star}\right) \\
& =\mathcal{L}_{A}^{\boldsymbol{h}}\left(\boldsymbol{\Lambda}_{\text {inv }}\right)=\mathcal{L}_{A}^{\boldsymbol{h}}\left(\boldsymbol{F}^{\star}\right) \\
& =-\boldsymbol{A}-\lambda_{\text {trace }}^{\star} \boldsymbol{I},
\end{aligned}
$$

We also have $\operatorname{Tr}\left[\boldsymbol{F}^{\star} \boldsymbol{A}\right]=-\sum_{k \neq \bar{k}} g_{k}^{\star} \operatorname{Tr}\left[\boldsymbol{F}^{\star} \mathcal{D}\left[\boldsymbol{L}_{k}\right]\left(\boldsymbol{F}^{\star}\right)\right]$, which concludes the proof.

Proposition 3: Let $\left(\boldsymbol{F}^{\star}, \boldsymbol{g}\right)$ satisfy the constraints of (11). Fix any $\bar{k} \in\left\{1, \ldots, K_{g}\right\}$. Let $\boldsymbol{h}$ be the gain vector characterized by $h_{k}=q_{k}$ for all $k \neq \bar{k}$ and $h_{\bar{k}}=q_{\bar{k}}+\delta g$ for some infinitesimal $\delta g>0$. Let $\boldsymbol{F}^{\boldsymbol{h}}$ be the traceless solution of $\mathcal{L}_{A}^{\boldsymbol{h}}\left(\boldsymbol{F}^{\boldsymbol{h}}\right)+\boldsymbol{A}-\operatorname{Tr}[\boldsymbol{A}] \boldsymbol{I}=0$. Then $\operatorname{Tr}\left[\boldsymbol{A} \boldsymbol{F}^{\boldsymbol{h}}\right] \leqslant \operatorname{Tr}\left[\boldsymbol{A} \boldsymbol{F}^{\star}\right]$.

Proof: Equality constraints (11b)-(11c) imply that $\boldsymbol{F}$ is a smooth function of the gain vector, thus we can consider the linearization $\boldsymbol{F}^{\boldsymbol{h}}=\boldsymbol{F}^{\star}+\delta \boldsymbol{F}$ for some differential $\delta \boldsymbol{F}$. They also imply that $\operatorname{Tr}[\delta \boldsymbol{F}]=0, \delta \boldsymbol{F}$ is Hermitian, and that the following equalities hold:

$$
\begin{aligned}
\sum_{k} g_{k} \mathcal{D}\left[\boldsymbol{L}_{k}\right]\left(\boldsymbol{F}^{\star}\right) & =\frac{1}{n} \operatorname{Tr}[\boldsymbol{A}] \boldsymbol{I}-\boldsymbol{A} \\
\sum_{k} h_{k} \mathcal{D}\left[\boldsymbol{L}_{k}\right]\left(\boldsymbol{F}^{\star}+\delta \boldsymbol{F}\right) & =\frac{1}{n} \operatorname{Tr}[\boldsymbol{A}] \boldsymbol{I}-\boldsymbol{A}
\end{aligned}
$$

Subtracting (14) from (15) yields:

$$
\mathcal{L}_{A}^{\boldsymbol{g}}(\delta \boldsymbol{F})=-\delta g \mathcal{D}\left[\boldsymbol{L}_{\bar{k}}\right]\left(\boldsymbol{F}^{\star}+\delta \boldsymbol{F}\right) .
$$

Premultiplying both sides of this expression by $\boldsymbol{F}^{\star}$ and taking the trace yields:

$$
\begin{aligned}
\operatorname{Tr}\left[\boldsymbol{F}^{\star} \mathcal{L}_{A}^{\boldsymbol{g}}(\delta \boldsymbol{F})\right]=- & \operatorname{Tr}\left[\delta g \boldsymbol{F}^{\star} \mathcal{D}\left[\boldsymbol{L}_{\bar{k}}\right] \boldsymbol{F}^{\star}\right] \\
& -\operatorname{Tr}\left[\delta g \boldsymbol{F}^{\star} \mathcal{D}\left[\boldsymbol{L}_{\bar{k}}\right](\delta \boldsymbol{F})\right] .
\end{aligned}
$$

By using identity $\operatorname{Tr}\left[\boldsymbol{X}_{1} \mathcal{D}\left[\mathcal{L}_{k}\right]\left(\boldsymbol{X}_{2}\right)\right]=\operatorname{Tr}\left[\boldsymbol{X}_{2} \mathcal{D}\left[\mathcal{L}_{k}\right]\left(\boldsymbol{X}_{1}\right)\right]$ for any matrices $\boldsymbol{X}_{1}, \boldsymbol{X}_{2}$ on the left-hand side of (16), and identity $\operatorname{Tr}\left[\boldsymbol{X \mathcal { D }}\left[\boldsymbol{L}_{k}\right](\boldsymbol{X})\right]=-\operatorname{Tr}\left[\left[\boldsymbol{X}, \boldsymbol{L}_{k}\right]\left[\boldsymbol{X}, \boldsymbol{L}_{k}\right]^{\dagger}\right] / 2$ for any Hermitian matrix $\boldsymbol{X}$ on the right-hand side of (16), and by observing that $\operatorname{Tr}\left[\delta \boldsymbol{F} \mathcal{L}_{A}^{\boldsymbol{g}}\left(\boldsymbol{F}^{\star}\right)\right]=-\operatorname{Tr}[\delta \boldsymbol{F} \boldsymbol{A}]$, it follows that:

$$
\begin{aligned}
\operatorname{Tr}[\boldsymbol{A} \delta \boldsymbol{F}]= & \frac{-\delta g}{2} \operatorname{Tr}\left[\left[\boldsymbol{F}^{\star}, \boldsymbol{L}_{\bar{k}}\right]\left[\boldsymbol{F}^{\star}, \boldsymbol{L}_{\bar{k}}\right]^{\dagger}\right] \\
& +\operatorname{Tr}\left[\delta g \boldsymbol{F}^{\star} \mathcal{D}\left[\boldsymbol{L}_{\bar{k}}\right](\delta \boldsymbol{F})\right] .
\end{aligned}
$$

For $\left[\boldsymbol{F}^{\star}, \boldsymbol{L}_{\bar{k}}\right] \neq 0$, choosing $\delta g$ small enough allows to dominate the term in $\delta g \delta \boldsymbol{F}$, we then have $\operatorname{Tr}[\boldsymbol{A} \delta \boldsymbol{F}]<0$ implying $\operatorname{Tr}\left[\boldsymbol{A} \boldsymbol{F}^{\boldsymbol{h}}\right]<\operatorname{Tr}\left[\boldsymbol{A} \boldsymbol{F}^{\star}\right]$. For $\left[\boldsymbol{F}^{\star}, \boldsymbol{L}_{\bar{k}}\right]=0$ and recalling that both $\boldsymbol{F}^{\star}$ and $\boldsymbol{L}_{\bar{k}}$ are Hermitian, the term in $\delta g \delta \boldsymbol{F}$ annihilates as well and we have $\operatorname{Tr}\left[\boldsymbol{A} \boldsymbol{F}^{\boldsymbol{h}}\right]=$ $\operatorname{Tr}\left[\boldsymbol{A F ^ { \star }}\right]$.

Altogether, we get the following result.

Corollary 1: Let Assumptions 1, 2 hold. Then the decoherence minimization problem (11) is solved by $\boldsymbol{g}^{\star}=\overline{\boldsymbol{g}}$.

Proof: From Proposition 2 and continuity of $\mathcal{L}_{A}^{g}$, either the value of $g_{k}$ does not matter or the optimum corresponds to $g_{k}$ taking its minimum or its maximum value. Proposition 3 further implies that having $g_{k}$ at its lower bound cannot correspond to an isolated minimum of the cost function. Thus 
the only remaining possibilities are that either the value of $g_{k}$ does not matter, or it must take its maximum value.

A possible physical justification of this result is discussed in the conclusion.

\section{A QUBIT AS ENVIRONMENT}

The qubit is the most basic quantum system. Nevertheless, it is an ubiquitous model for expressing the presence of defects in an environment, see e.g. [8], [7]. Furthermore, the effects of several such independent environment qubits just add up in the decoherence induced on a target system [6]. This motivates to investigate the effect of adjusting decoherence rates on a qubit as environment. The low dimensionality will also allow us to illustrate the case not covered so far, namely the effect of adjusting non-Hermitian decoherence channels individually.

\section{A. Analysis}

We consider the special case of an environment consisting of a qubit $\mathcal{H}_{A}=\mathbb{C}^{2}$ rotating around the $z$ axis with frequency $h_{z}$, subject to relaxation in a thermal environment and to the three Pauli Hermitian decoherence channels:

$$
\begin{gathered}
\mathcal{L}_{A}(\rho)=-i\left[h_{z} \boldsymbol{\sigma}_{z}, \rho\right]+\gamma_{-} \mathcal{D}\left[\boldsymbol{\sigma}_{-}\right](\rho)+\gamma_{+} \mathcal{D}\left[\boldsymbol{\sigma}_{+}\right](\rho) \\
+\gamma_{x} \mathcal{D}\left[\boldsymbol{\sigma}_{x}\right](\rho)+\gamma_{y} \mathcal{D}\left[\boldsymbol{\sigma}_{y}\right](\rho)+\gamma_{z} \mathcal{D}\left[\boldsymbol{\sigma}_{z}\right](\rho),
\end{gathered}
$$

where we assume that the gain vector $\boldsymbol{g}:=$ $\left(\gamma_{+}, \gamma_{-}, \gamma_{x}, \gamma_{y}, \gamma_{z}\right)^{\top}$ can be arbitrarily tuned within a compact and strictly positive interval $\Omega:=[\boldsymbol{g}, \overline{\boldsymbol{g}}]$, so as to guarantee time-scale separation of the dynamics (1). The qubit is weakly interacting with a target system via a single interaction Hamiltonian (10), and we will specifically consider two separate cases: $\boldsymbol{A}=a_{x} \boldsymbol{\sigma}_{x}+a_{y} \boldsymbol{\sigma}_{y}$ and $\boldsymbol{A}=a_{z} \boldsymbol{\sigma}_{z}$. In both cases the related operator $\boldsymbol{B}$ is left arbitrary.

Proposition 4: Consider a qubit auxiliary system (17) and single interaction Hamiltonian (10). Let $\Omega=[\boldsymbol{g}, \overline{\boldsymbol{g}}]$ be a compact and strictly positive interval of gains and denote with $\hat{\Omega}$ the set of vertices of $\Omega$, i.e. where each gain takes an extremal value. Then the decoherence on target is minimized by $\boldsymbol{g}^{\star} \in \hat{\Omega}$ in both cases $\boldsymbol{A}=a_{x} \boldsymbol{\sigma}_{x}+a_{y} \boldsymbol{\sigma}_{y}$ or $\boldsymbol{A}=a_{z} \boldsymbol{\sigma}_{z}$. Furthermore, if $h_{z}=0$, at least one gain takes its maximum at the optimum. In particular:

(i) if $\boldsymbol{A}=a_{x} \boldsymbol{\sigma}_{x}$, then $\left(\gamma_{x}^{\star}, \gamma_{y}^{\star}\right)=\left(\bar{\gamma}_{x}, \underline{\gamma}_{y}\right)$;

(ii) if $\boldsymbol{A}=a_{y} \boldsymbol{\sigma}_{y}$, then $\left(\gamma_{x}^{\star}, \gamma_{y}^{\star}\right)=\left(\underline{x}_{x}, \bar{\gamma}_{y}\right)$;

(iii) if $\boldsymbol{A}=a_{x} \boldsymbol{\sigma}_{x}+a_{y} \boldsymbol{\sigma}_{y}$ and $h_{z}=0$, then $\boldsymbol{g}^{\star}=\overline{\boldsymbol{g}}$.

Proof: Define:

$$
\begin{aligned}
& A_{11}(\boldsymbol{g}):=\frac{\gamma_{+}+\gamma_{-}}{4}+\gamma_{y}+\gamma_{z} \\
& A_{22}(\boldsymbol{g}):=\frac{\gamma_{+}+\gamma_{-}}{4}+\gamma_{x}+\gamma_{z} \\
& A_{33}(\boldsymbol{g}):=\frac{\gamma_{+}+\gamma_{-}}{2}+\gamma_{x}+\gamma_{y} .
\end{aligned}
$$

The fixed point $\bar{\rho}_{A}:=\left(\boldsymbol{I}+\bar{\rho}_{x} \boldsymbol{\sigma}_{x}+\bar{\rho}_{y} \boldsymbol{\sigma}_{y}+\bar{\rho}_{z} \boldsymbol{\sigma}_{z}\right) / 2$ of $\mathcal{L}_{A}(\cdot)$ has coordinates $\bar{\rho}_{x}=\bar{\rho}_{y}=0$ and:

$$
\bar{\rho}_{z}(\boldsymbol{g})=\frac{\gamma_{+}-\gamma_{-}}{2 A_{33}(\boldsymbol{g})}
$$

In order to minimize the decoherence on the target, we aim to minimize $\Re\left[\operatorname{Tr}\left[\boldsymbol{F} \bar{\rho}_{A} \boldsymbol{A}\right]\right]$ which reads as:

$$
\Re\left[\operatorname{Tr}\left[\boldsymbol{F} \bar{\rho}_{A} \boldsymbol{A}\right]\right]=\frac{A_{11} a_{x}^{2}+A_{22} a_{y}^{2}}{h_{z}^{2}+A_{11} A_{22}}+\frac{a_{z}^{2}\left(1-\bar{\rho}_{z}^{2}\right)}{A_{33}},
$$

where we drop the dependency on $\boldsymbol{g}$ for brevity and we took into account the fact that $\mathcal{L}_{A}\left(\boldsymbol{F} \bar{\rho}_{A}\right)+\boldsymbol{A} \bar{\rho}_{A}=\operatorname{Tr}\left[\boldsymbol{A} \bar{\rho}_{A}\right] \bar{\rho}_{A}$ and $\operatorname{Tr}\left[\boldsymbol{F} \bar{\rho}_{A}\right]=0$.

(Case $\left.\boldsymbol{A}=a_{x} \boldsymbol{\sigma}_{x}+a_{y} \boldsymbol{\sigma}_{y}\right)$. Let $s:=\left(\gamma_{+}+\gamma_{-}\right) / 4+\gamma_{z}$. Then (18) reads as:

$$
f(\boldsymbol{g})=\frac{1}{D(\boldsymbol{g})}\left(\left(s+\gamma_{y}\right) a_{x}^{2}+\left(s+\gamma_{x}\right) a_{y}^{2}\right),
$$

with $D(\boldsymbol{g}):=h_{z}+\left(s+\gamma_{x}\right)\left(s+\gamma_{y}\right)$. First, the partial derivative:

$$
\begin{aligned}
\frac{d f}{d s}=- & \frac{1}{D(\boldsymbol{g})^{2}}\left[\left(a_{x}^{2}+a_{y}^{2}\right) s^{2}+2\left(\gamma_{x} a_{y}^{2}+\gamma_{y} a_{x}^{2}\right) s\right. \\
& \left.+\left(\gamma_{x}+\gamma_{y}\right)\left(\gamma_{x} a_{y}^{2}+\gamma_{y} a_{x}^{2}\right)-h_{z}^{2}\left(a_{x}^{2}+a_{y}^{2}\right)\right]
\end{aligned}
$$

is either non-positive for all $s \in \mathbb{R}$ or is positive only in the interval $\left(s_{-}, s_{+}\right)$, with $s_{-}$being its negative root $s_{-}<$ $-\left(\gamma_{x} a_{y}^{2}+\gamma_{y} a_{x}^{2}\right) /\left(a_{x}^{2}+a_{y}^{2}\right)$ and $s_{+}$being the other one. Since $s$ must be chosen in a strictly positive interval, we can thus observe that, for any fixed $\gamma_{x}, \gamma_{y}$, the optimal $s$ must be at the extrema of its interval. Second, the partial derivative:

$$
\frac{d f}{d \gamma_{y}}=\frac{h_{z}^{2} a_{x}^{2}-s a_{y}^{2}\left(s+\gamma_{x}\right)}{D(\boldsymbol{g})^{2}}
$$

is sign-definite for any fixed $\gamma_{x}$ and $s$, and thus the optimal $\gamma_{y}$ must be at the extrema of its interval. Statement $\boldsymbol{g}^{\star} \in \hat{\Omega}$ then follows from symmetry of the $\gamma_{x}$ and $\gamma_{y}$ cases.

(Case $\boldsymbol{A}=a_{z} \boldsymbol{\sigma}_{z}$ ). We want to minimize (18) over $\boldsymbol{g}$. To this end, we treat $\gamma_{x}+\gamma_{y}$ as a single optimization variable $\gamma_{x y}$, and we introduce two new optimization variables $c_{+}, \gamma$ such that $\gamma_{+}=\gamma\left(1+c_{+}\right)$and $\gamma_{-}=\gamma$. Without loss of generality, it holds $c_{+} \geqslant 0$. Then (18) reads as:

$$
\begin{aligned}
f(\boldsymbol{g}) & =\frac{a_{z}^{2}}{D(\boldsymbol{g})^{3}}\left(\gamma_{+} \gamma_{-}+\gamma_{x y}^{2}+\left(\gamma_{-}+\gamma_{+}\right) \gamma_{x y}\right) \\
& =\frac{a_{z}^{2}}{D(\boldsymbol{g})^{3}}\left(\gamma_{x y}^{2}+\gamma\left(2+c_{+}\right) \gamma_{x y}+\gamma^{2}\left(1+c_{+}\right)\right),
\end{aligned}
$$

with $D(\boldsymbol{g}):=\gamma_{x y}+\left(\gamma_{-}+\gamma_{+}\right) / 2$. First, by considering the partial derivative:

$\frac{d f}{d \gamma_{x y}}=-\frac{a_{z}^{2}}{D(\boldsymbol{g})^{4}}\left(\gamma_{x y}^{2}+\gamma\left(2+c_{+}\right) \gamma_{x y}+\gamma^{2}\left(1+c_{+}-\frac{c_{+}^{2}}{2}\right)\right)$,

and computing its roots $\gamma_{x y, \pm}=\frac{\gamma}{2}\left(-2-(1 \mp \sqrt{3}) c_{+}\right)$, since the local minimum is achieved at $\gamma_{x y,-}<0$ and the local maximum is achieved at $\gamma_{x y,+} \geqslant \gamma_{x y,-}$ and $\gamma_{x y}$ must be chosen in a strictly positive interval, we observe that for any fixed $\gamma_{+}, \gamma_{-}$, the optimal $\gamma_{x y}$ is at the extrema of that interval. Second, by observing the sign of the partial derivative:

$$
\frac{d f}{d \gamma_{+}}=-a_{z}^{2} \frac{\gamma_{-}+\gamma_{x y}}{2 D(\boldsymbol{g})^{4}}\left(2 \gamma_{+}+\left(\gamma_{x y}-\gamma_{-}\right)\right)
$$

we observe that, for any fixed $\gamma_{x y}, \gamma_{-}$, the optimal $\gamma_{+}$must be at one extrema of its interval. Statement $\boldsymbol{g}^{\star} \in \hat{\Omega}$ then follows from symmetry of the $\gamma_{-}$and $\gamma_{+}$cases. 
Furthermore, if $h_{z}=0$, Proposition 1 implies that at least one optimal gain is at its maximum.

Case (i). From (22) with $a_{y}=0$, it is straightforward to compute: $d f / d \gamma_{y}=h_{z} a_{x}^{2} / D^{2} \geqslant 0$, which implies that, for any fixed $g$ and $\gamma_{x}$, the optimal $\gamma_{y}$ is $\underline{\gamma}_{y} ; d f / d \gamma_{x}=$ $-\left(\left(s+\gamma_{y}\right) a_{x} / D\right)^{2} \leqslant 0$, which implies that, for any fixed $g$ and $\gamma_{y}$, the optimal $\gamma_{x}$ is $\bar{\gamma}_{x}$.

Case (ii) follows by symmetry with (i).

Case (iii). If $h_{z}=0$, then $f(\boldsymbol{g})=a_{x}^{2} /\left(s+\gamma_{x}\right)+a_{y}^{2} /(s+$ $\left.\gamma_{y}\right)$. By monotonicity of $f(\boldsymbol{g})$ with respect to $\gamma_{x}, \gamma_{y}, s$, it follows that $\boldsymbol{g}^{\star}=\overline{\boldsymbol{g}}$.

\section{B. Simulations}

Section III-B states that, at least for a single Hamiltonian coupling, the gain on Hermitian decoherence channels must be maximized in order to minimize induced decoherence on target, while Proposition 4 cases (i),(ii) show that in presence of non-Hermitian decoherence channels this is not always true anymore. In this section, we directly investigate how the choice of optimal gains for the qubit example can depend on the tuning interval.

First, we consider the case of $\boldsymbol{A}=a_{x} \boldsymbol{\sigma}_{x}+a_{y} \boldsymbol{\sigma}_{y}$ and $h_{z} \neq 0$. According to the computations above, the induced decoherence $f\left(\gamma_{x}, \gamma_{y}, s\right)$ only depends on $\gamma_{x}, \gamma_{y}$ and $s=$ $\left(\gamma_{+}+\gamma_{-}\right) / 4+\gamma_{z}$. On Figure 1 (a) we show how the optimal gains depend on the lower bound for the tuning interval. When very low values are allowed for all the gains, the optimum is to put all gains at their minimum value; however, as soon as one of the decoherence channels is confined to larger values, the optimal situation is with all the gains at their maximum. The threshold between the two is a function of $h_{z}$. On Figure 1 (c), we investigate what happens when $s$ has a fixed value. For $s<s^{T H R}$, the optimal values of $\gamma_{x}$ and $\gamma_{y}$ are their minimum ones, while for $s>s^{T H R}$ they should be maximum. The value $s^{T H R}$ is a function of $a_{x} / a_{y}$ and of the upper bounds on the tuning intervals. Figure 1 (b) shows how the induced decoherence depends on $\gamma_{x}$ and $\gamma_{y}$, for values of $s$ above and below this threshold.

Second, we consider the case of $\boldsymbol{A}=a_{z} \boldsymbol{\sigma}_{z}$. Let $\gamma_{x y}:=$ $\gamma_{x}+\gamma_{y}$. Then the induced decoherence on target (18) simplifies to the function $f\left(\gamma_{-}, \gamma_{+}, \gamma_{x y}\right)$ in (22). On Figure 2 (a) we again show how the optimal gains depend on the lower bound for the tuning interval. The situation now is somewhat more complicated but can be understood on intuitive grounds. If $\gamma_{-}$is allowed to reach a very small value and $\gamma_{x y}$ is not constrained to too high values, then the best is to just push with $\gamma_{+}$, making the environment qubit converge essentially towards the pure state $|e\rangle\langle e|$. A symmetric situation is obtained when $\gamma_{+}$is allowed to take very small values. In all other cases, we should push all decoherence gains to the maximum. This behavior in the optimal gain selection is mirrored in Figure 2 (b), where we show how the optimum depends on the upper bound of the tuning interval. As soon as we can push $\gamma_{+}$at a value significantly larger than $\gamma_{-}$, we just do this and take $\gamma_{+}$ maximal and $\gamma_{-}$minimal; and conversely when the upper bound on $\gamma_{-}$is larger than the one on $\gamma_{+}$. When both $\gamma_{-}$and $\gamma_{+}$are restricted to a small interval of values, we are back to the situation where the optimum corresponds to making them all maximal. Finally, on Figure 2 (c) we show how the fixed value of $\gamma_{x y}$ influences the optimal choice. For $\gamma_{x y}$ below a threshold value that depends on the upper bounds of $\Omega$, the dominating effect of having only $\gamma_{-}$or only $\gamma_{+}$ is most beneficial, driving the qubit very close to a pure state $|g\rangle\langle g|$ or $|e\rangle\langle e|$ respectively; which one to choose is perfectly symmetric in this case. However, once $\gamma_{x y}$ exceeds the threshold value, the purifying effect appears to be not beneficial enough and the optimum instead corresponds to putting all decoherence gains to their maximum.

Note that all these conclusions are in agreement with the general statement of Proposition 1.

\section{CONCLUding Discussion}

We have studied the decoherence induced on a target quantum system by its weak coupling to a strongly dissipative environment. Under mild assumptions, this setting allows for a model reduction expressing the dominating decoherence induced on the target system by algebraic formulas. According to these formulas, the way to obtain as little as possible decoherence on the target system depends nontrivially on the environment parameters. In particular, we have investigated how it depends on the gains of various fixed decoherence channels acting on the environment. It appears, maybe counterintuitively, that having stronger mixing in the environment can induce less dissipation on the coupled target system; while in general the optimization of decoherence gains is a nontrivial problem depending on the available tuning intervals.

After reformulating the problem of minimizing the decoherence induced on the target system as a quadratic optimization problem on the variables associated with adiabatic elimination, we have proved two precise results. First, as a simple general observation, adiabatic elimination reverses the time scaling: the stronger the environment dissipates, the weaker the reduced-order model decoheres. Second, having only Hermitian decoherence channels in the environment, which thus cause its entropy to increase: decoherence induced on target is minimized by pushing each of these entropy-increasing channels in the environment to their maximal strength. Finally, we have explored a case with nonHermitian decoherence channels for an environment consisting of a single qubit. In this case a transition is observed between two behaviors: when gain choices are available which would stabilize the environment close to a pure state, this choice is taken; while if the environment steady state cannot be made sufficiently pure, then it is preferred again to push all gains to the maximum.

In all these results, we have never encountered a case where some decoherence gain should be chosen inside the optimization interval. This may be a property of the considered cases, or maybe more general. Therefore ongoing work is further focusing on studying non-Hermitian decoherence channels for general open quantum systems and interaction Hamiltonians consisting of multiple terms. Another major 

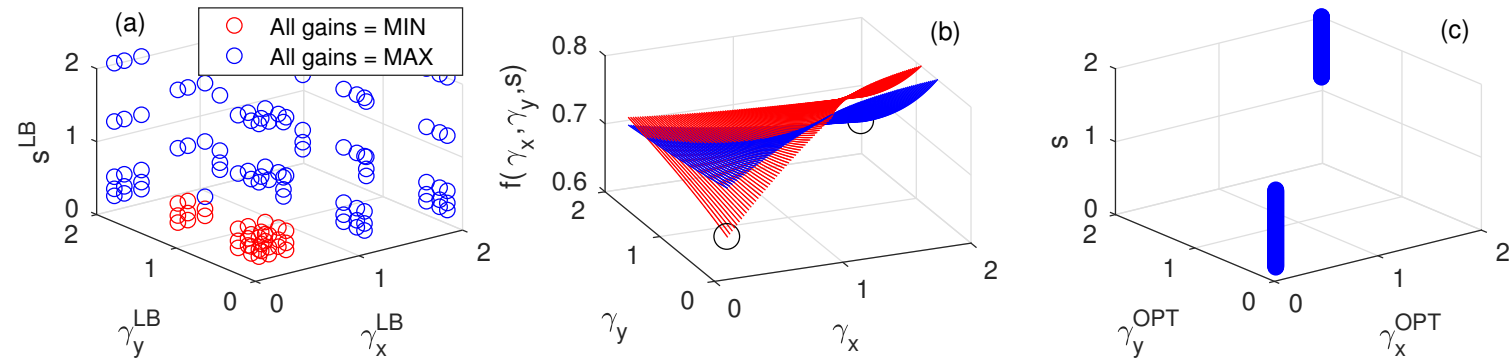

Fig. 1. Section IV, Proposition 4, case $\boldsymbol{A}=a_{x} \boldsymbol{\sigma}_{x}+a_{y} \boldsymbol{\sigma}_{y}$ and $h_{z} \neq 0$ : (a) Showing how the optimal decoherence gains depend on the lower bound $\left(s^{L B}, \gamma_{x}^{L B}, \gamma_{y}^{L B}\right)$ of the optimization interval, for a fixed $\bar{g}=[2,2,2]$. For each point $\boldsymbol{g}$ in the graph with coordinates $\left(s^{L B}, \gamma_{x}^{L B}, \gamma_{y}^{L B}\right)$, the optimal gain $\boldsymbol{g}^{\star} \in[\boldsymbol{g}, \overline{\boldsymbol{g}}]$ is evaluated and color-coded according to the legend; (b) two plots of $f\left(\gamma_{x}, \gamma_{y}, s\right)$ as in (19) respectively evaluated at $s_{1}=1$ (in red) and $s_{2}=1.4$ (in blue), together with their associated minima (in black circles); (c) for any given $s \in[0.1,2]$, the optimal gains $\gamma_{x}^{O P T}, \gamma_{y}^{O P T}$ are computed within the interval $[0.1,2] \times[0.1,2]$. Parameter values for simulations are: $a_{x}=1, a_{y}=1.4, h_{z}=2$.
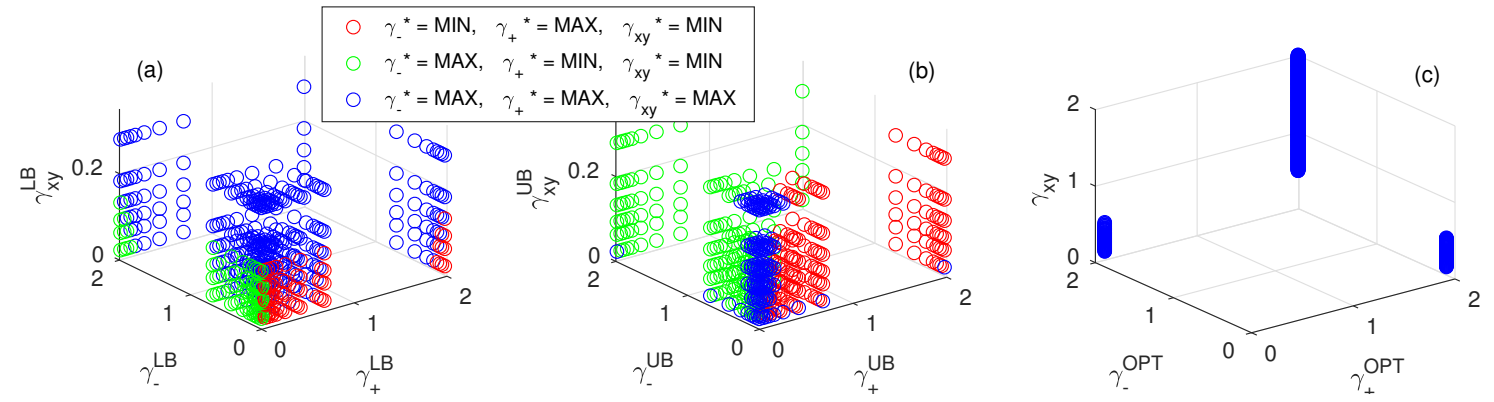

Fig. 2. Section IV, Proposition 4, case $\boldsymbol{A}=a_{z} \boldsymbol{\sigma}_{z}$ : (a) Showing how the optimal decoherence gains depend on the lower bound $\left(\gamma_{x y}^{L B}, \gamma_{-}^{L B}, \gamma_{+}^{L B}\right)$ of the optimization interval, for a fixed $\overline{\boldsymbol{g}}=[2,2,2]$. For each point $\boldsymbol{g}$ in the graph with coordinates $\left(\gamma_{x y}^{L B}, \gamma_{-}^{L B}, \gamma_{+}^{L B}\right)$, the optimal gain $\boldsymbol{g}^{\star} \in[\boldsymbol{g}, \overline{\boldsymbol{g}}]$ is evaluated and color-coded according to the legend; (b) Showing how the optimal decoherence gains depend on the upper bound $\left(\gamma_{x y}^{U B}, \gamma_{-}^{U B}, \gamma_{+}^{U B}\right)$ of the optimization interval, for a fixed $\boldsymbol{g}=[0.02,0.02,0.02]$. For each point $\overline{\boldsymbol{g}}$ in the graph with coordinates $\left(\gamma_{x y}^{U B}, \gamma_{-}^{U B}, \gamma_{+}^{U B}\right)$, the optimal gain $\boldsymbol{g}^{\star} \in[\boldsymbol{g}, \overline{\boldsymbol{g}}]$ is evaluated and color-coded according to the legend; (c) for any given $\gamma_{x y} \in[0.1,2]$, the optimal gains $\gamma_{+}^{O P T}, \gamma_{-}^{O P T}$ are computed within the interval $[0.1,2] \times[0.1,2]$

point for future work is to consider a different cost function, in a reservoir engineering context, where the goal on the target subsystem is to stabilize as strongly as possible a target subspace ("error-correcting-code-space") while minimizing any other decoherence effects.

We cannot close the paper without a tentative discussion of the somewhat counterintuitive conclusions that, the faster the environment dissipates, the slower the reduced-order model decoheres. One may want to connect this observation to classical mechanical systems. Indeed, for e.g. flexible links or the pendulum-on-cart system, by weakly coupling two degrees of freedom, it is often observed that the larger the dissipation on the first link is designed, the smaller the dissipation on the second is obtained. This analogy may be valid for quantum decoherence channels of the stabilizing type, e.g. $\boldsymbol{L}_{k}=|g\rangle\langle e|$. However, for $\boldsymbol{L}_{k}$ Hermitian, decoherence towards a state $\bar{\rho}_{A}$ of full rank means convergence towards a mixed, stochastic and possibly very dynamic situation whose expectation is given by $\bar{\rho}_{A}$. This dynamic stochastic situation does not appear to be as clearly linked to highly dissipative mechanical systems. One may thus rather have to view our results as saying that faster mixing in the environment leads to less uncertainty on the target system. This would be more reminiscent of an averaging-type interpretation, with fast motion of the environment weakening its coupling to the target system. In the same sense, the dynamical decoupling approach [10] explicitly aims at filtering out unwanted system-environment interactions by adding appropriate Hamiltonian drives that make the system "average out" the coupling Hamiltonian; the observations in our paper might be viewed as the stochastic equivalent of this.

\section{APPENDIX}

\section{A. Technical lemmas}

Lemma 1: If $\tilde{\boldsymbol{x}}^{\star}:=\left\{\boldsymbol{F}^{\star}, \boldsymbol{g}^{\star}, \lambda_{\text {trace }}^{\star}, \boldsymbol{\Lambda}_{\text {inv }}^{\star}, \boldsymbol{\mu}^{\star}, \overline{\boldsymbol{\mu}}^{\star}\right\}$ is a minimizer for (11), then:

(i) $\boldsymbol{\Lambda}_{i n v}^{\star}=\boldsymbol{F}^{\star}+f^{\star} \boldsymbol{I}$ for some $f^{\star} \in \mathbb{C}$;

(ii) $\operatorname{Tr}\left[\boldsymbol{\Lambda}_{i n v}^{\star} \mathcal{D}\left[\boldsymbol{L}_{k}\right]\left(\boldsymbol{F}^{\star}\right)\right] \leqslant 0$ for all $k \in\{1, \ldots, K\}$;

(iii) $\operatorname{Tr}\left[\boldsymbol{F}^{\star} \boldsymbol{A}\right]=-\sum_{k} g_{k}^{\star} \operatorname{Tr}\left[\boldsymbol{F}^{\star} \mathcal{D}\left[\boldsymbol{L}_{k}\right]\left(\boldsymbol{F}^{\star}\right)\right]$.

Proof: Taking the trace over equation (13a) yields $\lambda_{\text {trace }}^{\star}=-\operatorname{Tr}[\boldsymbol{A}] / n$, which implies that (13a) and (11c) are the same equation, and thus (i). It follows that:

$$
\begin{aligned}
\operatorname{Tr} & {\left[\boldsymbol{\Lambda}_{i n v}^{\star} \mathcal{D}\left[\boldsymbol{L}_{k}\right]\left(\boldsymbol{F}^{\star}\right)\right]=\operatorname{Tr}\left[\boldsymbol{F}^{\star} \mathcal{D}\left[\boldsymbol{L}_{k}\right]\left(\boldsymbol{F}^{\star}\right)\right] } \\
& =-\frac{1}{2} \operatorname{Tr}\left[\boldsymbol{S}_{k}^{\dagger} \boldsymbol{S}_{k}\right] \leqslant 0, \quad \forall k \in\{1, \ldots K\},
\end{aligned}
$$

with $\boldsymbol{S}_{k}:=\left[\boldsymbol{F}^{\star}, \boldsymbol{L}_{k}\right]$. Furthermore, by multiplying both sides of (13a) by $\boldsymbol{F}^{\star}$ and taking the trace again, we get (iii). 
Lemma 2: If $\tilde{\boldsymbol{x}}^{\star}:=\left\{\boldsymbol{F}^{\star}, \boldsymbol{g}^{\star}, \lambda_{\text {trace }}^{\star}, \boldsymbol{\Lambda}_{\text {inv }}^{\star}, \boldsymbol{\mu}^{\star}, \overline{\boldsymbol{\mu}}^{\star}\right\}$ is a minimizer for (11) and $\operatorname{Tr}\left[\boldsymbol{F}^{\star} \mathcal{D}\left[\boldsymbol{L}_{\bar{k}}\right]\left(\boldsymbol{F}^{\star}\right)\right]=0$ for some $\bar{k}$, then:

(i) $\left[\boldsymbol{L}_{\bar{k}}, \boldsymbol{F}^{\star}\right]=0$;

(ii) $\mathcal{D}\left[\boldsymbol{L}_{\bar{k}}\right]\left(\boldsymbol{F}^{\star}\right)=0$;

(iii) $\operatorname{Tr}\left[\boldsymbol{F}^{\star} \boldsymbol{A}\right]=-\sum_{k \neq \bar{k}} g_{k}^{\star} \operatorname{Tr}\left[\boldsymbol{F}^{\star} \mathcal{D}\left[\boldsymbol{L}_{k}\right]\left(\boldsymbol{F}^{\star}\right)\right]$.

Proof: By making use of (23), we have $\operatorname{Tr}\left[\boldsymbol{F}^{\star} \mathcal{D}\left[\boldsymbol{L}_{\bar{k}}\right]\left(\boldsymbol{F}^{\star}\right)\right]=-\operatorname{Tr}\left[\left[\boldsymbol{F}^{\star}, \boldsymbol{L}_{\bar{k}}\right]^{\dagger}\left[\boldsymbol{F}^{\star}, \boldsymbol{L}_{\bar{k}}\right]\right] / 2=0$ which implies (i). It thus follows that $\mathcal{D}\left[\boldsymbol{L}_{\bar{k}}\right]\left(\boldsymbol{F}^{\star}\right)=0$ which, together with statement (iii) in Lemma 1, implies (iii).

\section{REFERENCES}

[1] Rémi Azouit, Francesca Chittaro, Alain Sarlette, and Pierre Rouchon. Towards generic adiabatic elimination for bipartite open quantum systems. Quantum Science and Technology, 2(4):044011, 2017.

[2] J. Carr. Applications of Centre Manifold Theory. Applied Mathematical Sciences. Springer New York, 2012.

[3] Man-Duen Choi. Completely positive linear maps on complex matrices. Linear Algebra and its Applications, 10(3):285 - 290, 1975.
[4] Joachim Cohen. Autonomous quantum error correction with superconducting qubits. PhD thesis, PSL Research University, 2017.

[5] Neil Fenichel. Geometric singular perturbation theory for ordinary differential equations. Journal of Differential Equations, 31(1):53 98, 1979.

[6] P. Forni, A. Sarlette, T. Capelle, E. Flurin, S. Deléglise, and P. Rouchon. Adiabatic elimination for multi-partite open quantum systems with non-trivial zero-order dynamics. In 2018 IEEE Conference on Decision and Control (CDC), pages 6614-6619, Dec 2018.

[7] Jiansong Gao, Miguel Daal, John M. Martinis, Anastasios Vayonakis, Jonas Zmuidzinas, Bernard Sadoulet, Benjamin A. Mazin, Peter K. Day, and Henry G. Leduc. A semiempirical model for two-level system noise in superconducting microresonators. Appl. Phys. Lett., 92:212504, May 2008.

[8] Jürgen Lisenfeld, Grigorij J. Grabovskij, Clemens Müller, Jared H. Cole, Georg Weiss, and Alexey V. Ustinov. Observation of directly interacting coherent two-level systems in an amorphous material Nature Communications, 6, Feb 2015.

[9] J. F. Poyatos, J. I. Cirac, and P. Zoller. Quantum reservoir engineering with laser cooled trapped ions. Phys. Rev. Lett., 77:4728-4731, Dec 1996.

[10] Lorenza Viola, Emanuel Knill, and Seth Lloyd. Dynamical decoupling of open quantum systems. Physical Review Letters, 82(12):2417, 1999. 